\title{
Would delaying the school day prevent anxiety in adolescents?
}

\author{
By Dr. Jessica K Edwards
}

Stressful life events (SLEs) are common during adolescence and can leave some individuals vulnerable to developing internalizing symptoms., ${ }^{1,2}$ Despite the recognition of this association, little is known about the underlying mechanisms. A new study published in the Journal of Child Psychology and Psychiatry has investigated whether sleep duration and regularity might link SLEs to anxiety and depression symptoms. "Due to a series of puberty-driven changes in homeostatic and circadian systems, as well as socio-cultural factors that are part of typical development, adolescents tend to start going to bed later than they did as children", explains lead author Constanza Vidal Bustamante. "However, their wake-up time remains fixed due to early school start times, leaving most adolescents sleep deprived".

Much research has investigated adolescent stress, sleep, and anxiety and depression symptoms via self-report over short periods. This approach can be prone to recall biases ${ }^{3}$ and fails to capture changes within individuals over time. ${ }^{4}$ To overcome this issue, the researchers followed a group of 30 girls (aged 15-17 years old) for a full year. They used Fitbits to track their sleep passively and continuously throughout the study period. The participants also completed a series of evaluations assessing SLEs and anxiety and depression symptoms once per month. 
